\title{
Investigation of Dynamics of Damped Bearing-Rotor System of Centrifugal Milk Separator
}

\author{
Andrius TRUMPA, Vadim MOKŠIN*, Vytautas TURLA, Nikolaj ŠEŠOK, Igor ILJIN
}

\begin{abstract}
This paper analyses the dynamic behaviour of centrifugal milk separator's bearing-rotor system equipped with vibration absorbers. It is shown that rotational milk motion inside the separator causes a centrifugal force that increases dynamic unbalance of the rotor and has a negative effect on performance of the bearings. Mathematical simulation of bearing-rotor system has shown that the rotor rotates at frequency that is outside the resonant frequency region and the amplitude resonances are associated with the bearings and the components of step-up gear. Investigations of vibration transmissibility of vibration damping system have shown that the resonant frequencies are outside the rotor resonance interval and damping system suppresses the effect of separator with milk on rotor bearings and housing. However, it distorts results of diagnostic measurements. Experimental studies confirmed the last statement and showed that results of indirect (through the housing) vibration measurements cannot be used to diagnose the state of damped bearing-rotor system.
\end{abstract}

Keywords: bearing-rotor system; centrifugal milk separator; damping; fault diagnostics; unbalance

\section{INTRODUCTION}

Usually, the dynamic models are developed to investigate the dynamic response of rotors. Dynamic model shall include mass and stiffness of the rotor and supports as well as the damping of the supports. Forces caused by unbalance or centrifugal forces are considered as the excitation forces. Bearing-rotor system of modern centrifugal milk separator requires precise and reliable fault diagnostics $[1,2]$. The centrifugal effect of moving mass (milk), motor, gearbox and the unbalance of the rotor are the main sources of vibration in centrifugal separators. It is practically impossible to balance rotating parts perfectly. Even though the perfect balance is achieved, it would be impossible to maintain it during operation for a variety of factors, such as thermal distortion, bearing jams, different thermal expansion of the parts, and so on.

In order to minimize vibrations, machines are modelled, then numerically simulated. It makes possible to change their parameters, reduce maintenance costs and save time. Modelling helps predict potential system failures and prevent their consequences. Fault detection and diagnosis techniques allow to monitor some important system parameters like pressure, temperature, vibration level and generate alarms if certain limits are exceeded, [3-7]. By applying static and dynamic process models, common process input and output measurements, the inherent relationships and redundancies can be used to detect faults earlier and localize them better [7]. The residual generation problem can be stated by considering a dynamic system with a known nominal mathematical model [7].

Unbalance of the rotor of centrifugal milk separator changes the dynamic behaviour of the system. Harsha [8] was the first who investigated the influence of unbalance forces on dynamic response of the rotor supported by cylindrical roller bearings that have eight rollers. Period doubling and mechanism of intermittency were observed that lead to chaos. Non-linear dynamic behaviour of unbalanced horizontal rigid rotor supported by deep groove ball bearings SKF6002 was studied by Tiwari et al. [9]. It was studied both numerically and experimentally. It was concluded that multi-frequency excitation due to varying compliance and unbalance force results in a response which has regions of instability and chaos. Frequency spectra displayed multiples of rotor rotating frequency and varying compliance frequency (the ball passage frequency) and the linear combination of two frequencies.

Upadhyay et al. [10] particularly investigated the dynamic behaviour of an unbalanced rotor supported by roller bearings with different radial internal clearance. The particular attention was focused on the non-linearity in the dynamic behaviour introduced by clearance. The regions of periodic, quasi-periodic and chaotic motion for ball bearing were bounded as an outcome of this work. Sunnersjo [11] investigated the varying complianceinduced vibrations theoretically and experimentally, taking damping and inertia forces into account. Fukata et al. [12] investigated compliance-induced vibrations and non-linear dynamic response of the ball bearing supporting a balanced horizontal rotor. A more detailed analysis was performed comparing with the Sunnersjo work [11] as regimes of subharmonic, super-harmonic and chaotic behaviour were obtained.

The varying compliance effect was investigated theoretically by Perret [13]. Elastic deformation between balls and race of a deep groove ball bearing was modelled by Hertzian theory. Meldau [14] investigated theoretically 2D motion of shaft centre. Both authors [13, 14] performed a quasi-static analysis since damping and inertia forces were not considered. Authors of the work [15] have developed a theoretical model of a balanced horizontal rigid rotor supported by ball bearings. A constant vertical load was applied to the system. Similar work was done by Fukata et al. [12], but more results were reported and two different routes to chaos were described in work done by Mevel and Guyader [15]. Chaos was reported to come out of quasi-periodic route and sub-harmonic route. Authors [16] developed a non-linear model for structural vibrations in the rolling bearing by considering the stiffness in contact between the rolling elements and races. However, distributed defects were not considered in this model. The effect of the radial bearing clearance on the dynamics of a balanced, horizontal rotor was studied in the work done by Tiwari et al. [17]. The appearance of sub-harmonics and Hopf bifurcation was observed theoretically whereas the shift in the peak response was also observed experimentally. 
Non-linear behaviour of a high-speed balanced rotor supported by ball bearings was studied in the work [18]. It was established that the most severe vibrations occur when the varying compliance frequency and its harmonics coincide with natural frequency. The effect of radial internal clearance and rotor speed was investigated by Harsha [19]. The appearance of chaotic, periodic, subharmonic and Hopf bifurcation was observed theoretically. In the analytical formulation the contact between the balls and races was considered as non-linear springs, whose stiffness was obtained by using Hertzian elastic contact deformation theory. Harsha [20] also studied the effects of rotor speed with geometrical imperfections of surface of the bearing components. The results were obtained from a large number of numerical integrations and were mainly presented in the form of Poincare maps and frequency spectra.

Yadav et al. [21] proposed to study the unbalanced rotor along with the internal radial clearance as nonlinearity.

In order to study the vibrational characteristics of rolling-element bearing, a model of bearing assembly can be considered as a spring-mass-damper system. It is assumed that there is a non-linear relationship between force and deformation of the race and balls. This relationship can be obtained by Hertzian theory.
The effect of unbalance load on sub-harmonic resonance of a ball bearing-rotor system is minor [22]. However, the response amplitude and sub-harmonic component of the rotor system rise significantly as the moment of couple increases [22]. It can be noted, that moment of couple has an impact influence on the subharmonic resonances of the ball bearing-rotor systems [22]. However, scientific literature provides no information regarding preloaded double-row bearings that are used in rotary milk processing equipment equipped with vibration suppression systems.

The main objective of this work is to demonstrate the problems appearing during diagnosis of the faults of damped bearing-rotor system that is used in milk separators and other rotating machinery used in dairy industry. In order to achieve the objective, the dynamic model was developed and numerical simulation was carried out. Due to the complexity of the problem and lack of scientific literature regarding simulation of such type systems, the experimental diagnostic measurements were carried out in which bearing vibrations were measured directly and through the housing. In order to improve reliability of fault diagnosis results it was suggested to attach an accelerometer directly to the outer ring of the bearing via rigid connector or in other words, to use direct bearing vibration measurement technique. However, the sufficient changes in design should be made in this case.

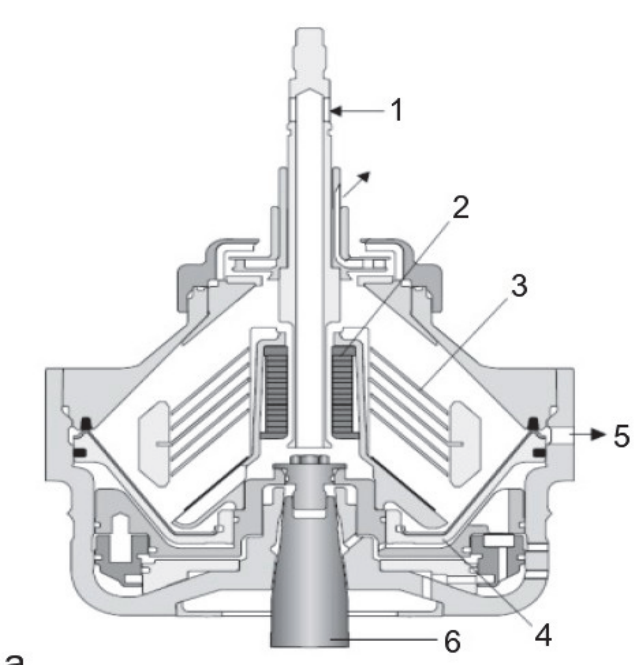

a

\section{Hysteresis damper}

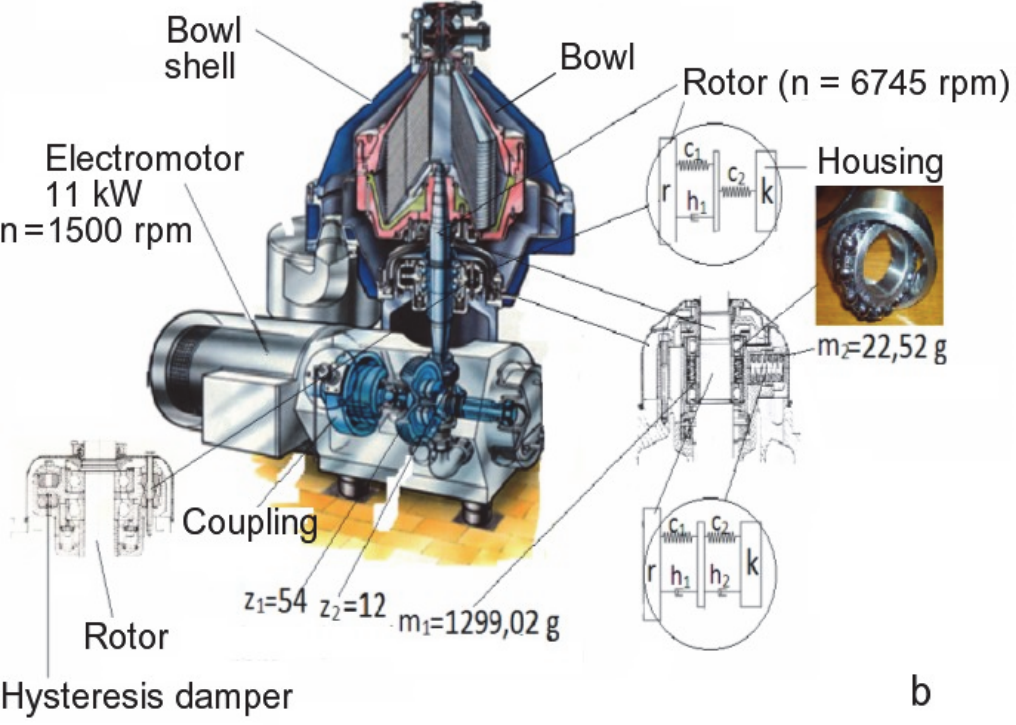

b

\section{OBJECT OF THE RESEARCH}

Scheme of the centrifugal milk separator is presented in Fig. 1a. The separation process takes place inside the bowl (Fig. 1b) which creates centrifugal forces and increases the unbalance of the rotor. The milk is fed into the rotating bowl through the feeding pipe 1 (Fig. 1a). Liquid is accelerated in the distributor 2 , before it enters the disc stack 3. Milk is introduced into separation channels at the outer edge of the disc stack 3 and flows inwards. On the way through the channels, solid particles are separated from the milk and thrown back along the undersides of the discs to the periphery of the separator bowl. Particles are accumulated in the solids space 4 and are automatically discharged at pre-set intervals through peripheral channels
5. The cream is less dense than the skim milk and therefore settles inwards in the channels towards the axis of rotation and passes to an axial outlet. The skim milk moves outwards to the space outside the disc stack and then through a channel between the top of the disc stack and the conical hood of the separator bowl.

Thus, separation process affects the dynamic behaviour of the rotor 6 (Fig. 1a) and especially of its rolling-element bearings. An effective method of reducing bearing vibrations is to provide vibration dampers in bearing units (Fig. 1b). Passive vibration adsorbers usually are designed to damp vibrations of higher frequencies in cases where high damping level is needed. They can work effectively only when external excitation frequency is significantly greater than their natural frequency. Under 
real conditions, however, excitation frequency is often variable over time either excitation contains a range of frequencies. As a result, external excitation frequencies can approach in magnitude natural frequencies, and effectiveness of passive adsorber can be severely reduced.

Bearing-rotor systems equipped with vibration adsorbers (Fig. 1b) are very common in dairy industry machinery. As their rotational speed and rotation accuracy are constantly increased, noise and vibration problems related to these systems are becoming more and more important [23]. Since rolling-element bearings are one of the biggest sources of noise and vibrations, they received a lot of attention as they significantly affect dynamic properties of rotor systems. This effect occurs for various reasons such as clearances between bearing elements, Hertzian forces, variable compliances, etc. [22]. Therefore, interest in analysis of vibrations of nonlinear rotor systems is constantly increased [22]. Rolling-element bearings exhibit very complex dynamic behaviour because of the large number of rolling elements. Kinematical behaviour is also very complex and depends on initial conditions. Shaft is supported at both ends by two rolling-element bearings, further the bowl is attached to its top (Fig. 1b). Motion of the liquid inside the bowl creates the centrifugal force that changes direction as the angular position of the rotor changes. Therefore, the dynamics of the whole system constantly changes [21].

Rolling-element bearings are the most vulnerable components of centrifugal milk separator shown in Fig. 1. In terms of fault diagnosis, this bearing-rotor system is difficult to diagnose as it is not possible to measure its vibrations due to the interference of vibration suppression system, which influences measurement results $[1,2]$. One of the most important tasks of diagnostic simulation is the modelling of real dynamic systems using mathematical models, robust algorithms and computer software. One such dynamic system is bearing-rotor system with vibration dampers whose fault diagnosis is complicated and requires specific modelling technique.

\section{NUMERICAL ANALYSIS OF MILK FLOW INSIDE THE BOWL}

SolidWorks 2011 SP2.0 and SolidWorks Flow Simulation 2011 software were used for analysis of liquid flow inside the bowl of centrifugal milk separator.

Numerical simulation was carried out by solving the Navier-Stokes equations that relate momentum, energy conservation and mass laws for fluid flow. Flow Simulation software utilizes Favre-averaged NavierStokes equations in which time-averaged effects of the turbulence on the flow parameters are considered, whereas the other, i.e., time-dependent, large-scale phenomena are considered directly [24, 25]. During this process, some extra terms known as the Reynolds stresses appear in the equations. To close this system of equations, transport equations for the turbulent kinetic energy and its dissipation rate have to be employed (so-called $k-\varepsilon$ model $[24,25])$.

Flow Simulation uses one system of equations to describe both turbulent and laminar flow. Moreover, transitions from a turbulent to laminar state and/or vice versa are possible [24].
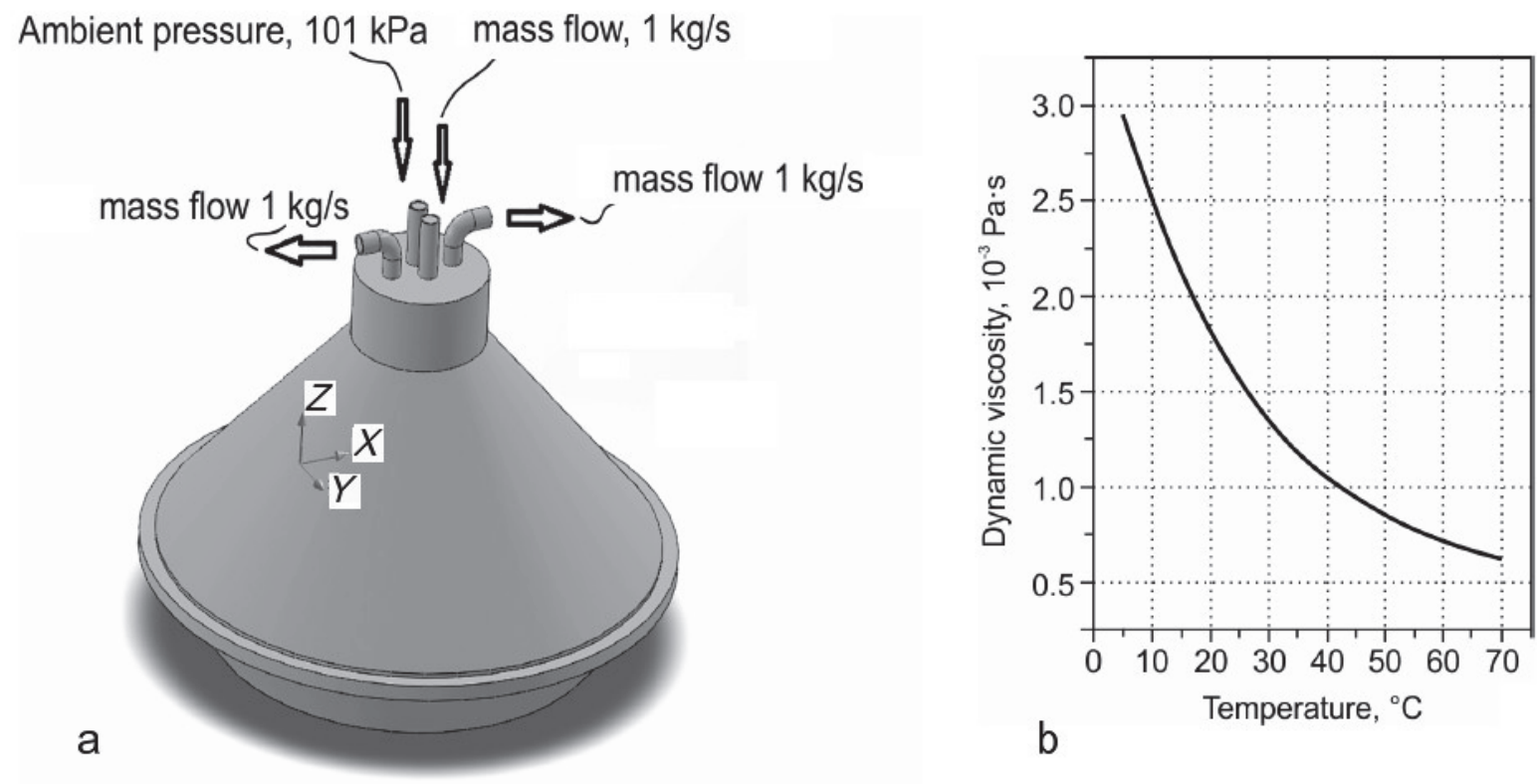

Figure 2 3D model of the bowl (a) and milk viscosity versus temperature curve (b)

$3 \mathrm{D}$ model of the bowl is shown in Fig. 2a. The following boundary and initial conditions were applied to the model: thermodynamic parameters (milk temperature $293.16 \mathrm{~K}$, viscosity $1.79 \cdot 10^{-3} \mathrm{~Pa} \cdot \mathrm{s}$ (Fig. $2 \mathrm{~b}$ ), and pressure $101325 \mathrm{~Pa}$ ), fluid inflow and outflow parameters (Fig. 2a). Milk temperature was not varied during the simulation. Geometric parameters of the bowl (Fig. 1) were as follows (Fig. 2a): height of the bowl was $580 \mathrm{~mm}$, biggest diameter of the bowl was $550 \mathrm{~mm}$, diameter of the inlet pipe was 10 $\mathrm{mm}$, diameter of the outlet pipe was $25 \mathrm{~mm}$.

Milk dynamic viscosity versus temperature curve is presented in Fig. 2b. Other simulation parameters were: the maximum number of time steps was 4000 , level of initial mesh (controls the resolution of the geometry by the mesh) was set to the maximum level 8, and result resolution level (governs the solution accuracy that can be interpreted as 
resolution of calculation results) was set to the maximum level 8 .

\section{RESULTS AND DISCUSSION \\ 4.1 Milk Flow Simulation}

Liquid flow trajectories are presented in Fig. 3. It can be seen from Fig. 3 that the flow moves down toward the bottom of the bowl due to the action of gravity and pressure. Then liquid is raised by rotating discs which separate solid particles from the pure milk. Then liquid exits the separator through the outlet pipes. The centrifugal force can be calculated using the following formula: $F_{\text {cen }}=\left(m v^{2}\right) / r$, where $m$ is the mass of the liquid, $v$ is the velocity, $r$ is the radius. Centrifugal force varies approximately from 15 to $58 \mathrm{~N}$.

Milk flow causes the centrifugal force in rotor system and the unbalance of the rotor is one of the main sources of vibrations. The unbalance changes the dynamical behaviour of the system. Formulas of rotor unbalance forces acting in direction of the $X$ and $Y$ axes (Fig. 2a) have the following appearance:

$$
\begin{aligned}
& F_{X}=m_{\mathrm{s}} e \omega^{2} \cos \omega t, \\
& F_{Y}=m_{\mathrm{s}} e \omega^{2} \sin \omega t
\end{aligned}
$$

where $m_{\mathrm{s}}$ is the mass of the bowl, $e$ is the distance between unbalance and the axis of rotation, $\omega$ is the angular velocity of the rotor, $t$ is the time.

Using the simulation, it is possible to evaluate the influence of unbalance forces on dynamical behaviour of bearing-rotor system. Eccentricity or unbalance values should be known. In our case, bowl mass eccentricity of 5 $\mu \mathrm{m}$ results in the resultant force of $319.21 \mathrm{~N}$ (Eq. (1)). Besides that, the milk flow inside the bowl creates centrifugal force which amplifies the effect of unbalance and directly affects the vibration dampers and the housing of the separator. Bearing life decreases and the reliability of diagnostic measurements reduces as a result.

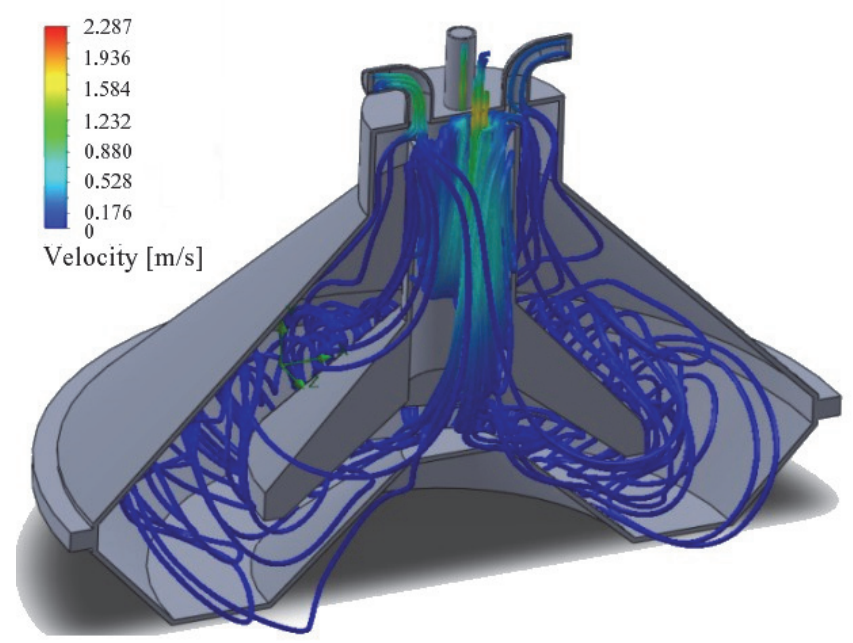

Figure 3 Milk flow trajectories inside the bowl of the centrifugal milk separator

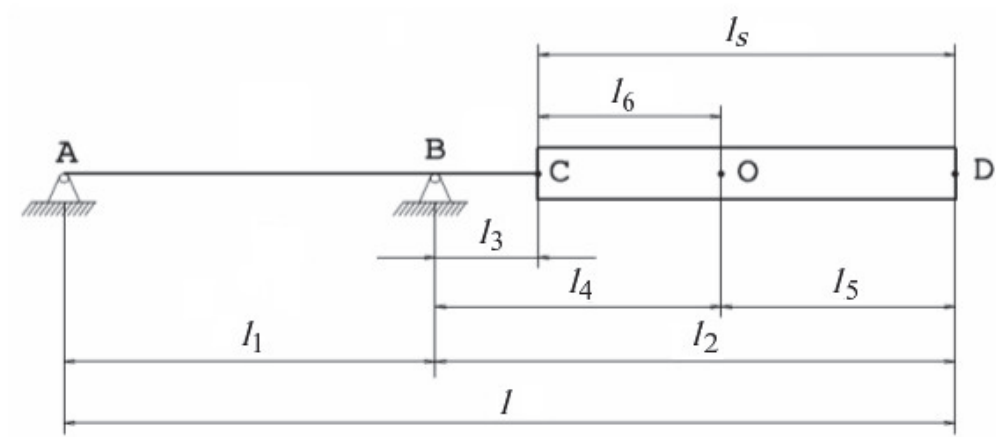

a

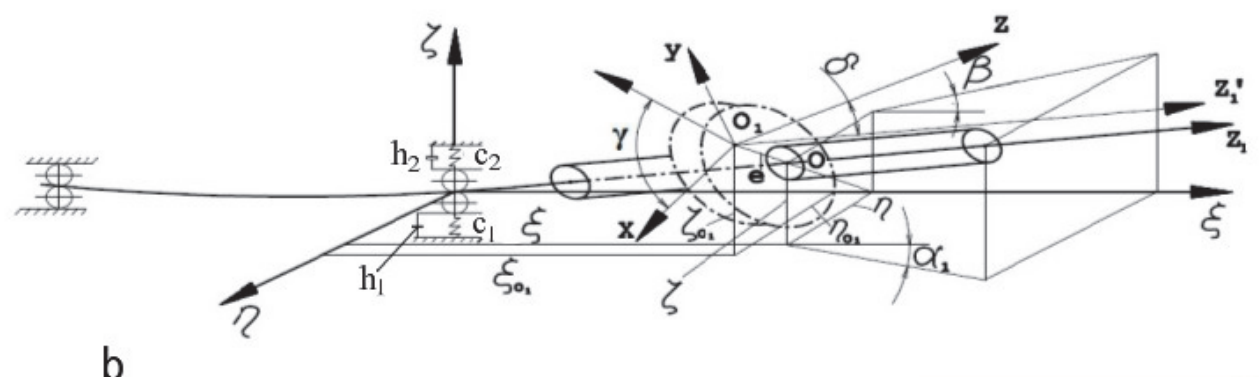

Figure 4 (a) Geometric parameters and (b) model of the bearing-rotor system of the separator 


\subsection{Modelling of the Bearing-Rotor System}

A model of the bearing-rotor system of the separator is presented in Fig. 4b. Let us consider the influence of unbalance on vibrations of the rotor. Let us introduce the fixed coordinate system $\xi \eta \zeta$ whose origin is located in the geometrical centre of upper bearing (Fig. 4b). $\xi$ axis coincides with the axis of the bearing. Let us introduce also coordinate system $x y z$, the origin of which coincides with centre of gravity $\mathrm{O}_{1}$ of the bowl. Coordinate axis $z_{1}$ of the bent rotor is parallel to the coordinate axis $z_{1}^{\prime}$ that passes through the origin $\mathrm{O}_{1}$. There is an angle $\delta$ between the axes $z$ and $z_{1}^{\prime}$ (Fig. 4 b).

The eccentricity $e$ (Fig. 4b) characterizes the static unbalance and the angle $\delta$ represents the instantaneous component of unbalance. Geometrical centre of the bowl is marked as point $\mathrm{O}$ (Fig. 4a, b). Position of the separator is described by the linear coordinates $\xi, \eta, \zeta$ of point $\mathrm{O}$ and by the angles $\alpha_{1}, \beta$ and $\varphi=\omega t$, where $\omega$ is the angular velocity of the rotor.

Then the system of differential equations of vibrations of the rotor will look like this:

$$
\begin{aligned}
& m_{\mathrm{s}} \ddot{\eta}+a_{1} \eta-a_{2} \alpha_{1}=m_{\mathrm{s}} e \omega^{2} \cos \omega t, \\
& m_{\mathrm{s}} \ddot{\zeta}+a_{1} \zeta-a_{2} \beta=m_{\mathrm{s}} e \omega^{2} \sin \omega t, \\
& I_{\mathrm{T}} \ddot{\beta}-I_{\mathrm{z}} \omega \dot{\alpha}_{1}-a_{2} \zeta+a_{3} \beta= \\
& -\left(I_{\mathrm{z}}-I_{\mathrm{T}}\right) \delta \omega^{2} \cos (\omega t-\gamma), \\
& I_{\mathrm{T}} \ddot{\alpha}_{1}+I_{\mathrm{z}} \omega \dot{\beta}-a_{2} \eta+a_{3} \alpha_{1}= \\
& \left(I_{\mathrm{z}}-I_{\mathrm{T}}\right) \delta \omega^{2} \sin (\omega t-\gamma),
\end{aligned}
$$

where

$$
\begin{aligned}
& a_{1}=\delta_{22} / \Delta^{2}, a_{2}=\delta_{12} / \Delta^{2}, a_{3}=\delta_{11} / \Delta^{2}, \Delta^{2}=\delta_{11} \delta_{22}-\delta_{12}^{2}, \\
& \delta_{11}=\frac{1}{c_{1}}\left(\frac{l_{4}}{l_{1}}\right)^{2}+\frac{1}{c_{2}}\left(\frac{l_{1}+l_{4}}{l_{1}}\right)^{2}+\frac{l_{1} l_{4}^{2}}{3 E I_{1}}+\frac{l_{4}^{3}-l_{6}^{3}}{3 E I_{2}}
\end{aligned}
$$

is the displacement of the point $\mathrm{O}$ due to the action of an unitary force applied to the same point [26], $\delta_{12}=\delta_{21}=\frac{1}{c_{1}} \frac{l_{4}}{l_{1}^{2}}+\frac{1}{c_{2}}\left(\frac{l_{1}+l_{4}}{l_{1}^{2}}\right)+\frac{l_{1} l_{4}}{3 E I_{1}}+\frac{l_{4}^{2}-l_{6}^{2}}{2 E I_{2}} \quad$ is the rotation angle of section passing through the point $\mathrm{O}$ caused by unitary force applied to the same point [26], $\delta_{22}=\left(\frac{1}{c_{1}}+\frac{1}{c_{2}}\right) \frac{1}{l_{1}^{2}}+\frac{l_{1}}{3 E I_{1}}+\frac{l_{4}-l_{6}}{E I_{2}}$ is the rotation angle of section passing through the point $\mathrm{O}$ caused by unitary moment [26], $I_{1}, I_{2}$ are the moments of inertia of rotor sections $\left(I_{1,2}=0.05 d^{4}\right), c_{1}, c_{2}$ are the stiffness of the bearing and the damping system, $t$ is the time, $m_{\mathrm{s}}$ is the mass of the bowl, $\omega$ is the angular velocity of the rotor, $I_{z}$ is the longitudinal moment of inertia of the bowl, $I_{\mathrm{T}}$ is the centroidal moment of inertia of the bowl.

Critical (resonant) angular velocities of the rotor can be established as the roots of characteristic equation:

$$
\Delta^{2} m_{\mathrm{s}}\left(I_{\mathrm{z}}-I_{\mathrm{T}}\right) \omega^{2}+\left[\delta_{11} m_{\mathrm{s}}-\delta_{22}\left(I_{\mathrm{z}}-I_{\mathrm{T}}\right)\right] \omega^{2}-1=0 .
$$

Then:

$\omega_{i}=\left\{-b+(-1)^{i}[b+4 a]^{1 / 2} / 2 a\right\}^{1 / 2}, i=1,2$

where $b=\delta_{11} m_{\mathrm{s}}-\delta_{22}\left(I_{\mathrm{z}}-I_{\mathrm{T}}\right), a=\Delta^{2} m_{\mathrm{S}}\left(I_{\mathrm{z}}-I_{\mathrm{T}}\right)$.

Rotor has only one critical angular velocity $\omega_{2}=1388.76 \mathrm{rad} / \mathrm{s}$.

Simulation and analysis of the model were carried out using Simulink and MATLAB software (The MathWorks, Inc., Natick, Massachusetts, USA). With reference to Eq. (2) and coefficients given in Tab. 1, dynamic model of bearing-rotor system was developed in Simulink environment. The $\eta$ coordinate axis was extremely important for the analysis because this axis coincides with the vibration measuring direction during fault diagnosis of

\begin{tabular}{|c|c|c|c|c|c|c|c|}
\hline Parameter & $m_{\mathrm{s}}$ & $I_{\mathrm{T}} / l^{2}$ & $I_{z} / l^{2}$ & $h_{1}$ & $h_{2}$ & $c_{1}$ & $c_{2}$ \\
\hline Dimension & $\mathrm{kg}$ & $\mathrm{kg}$ & $\mathrm{kg}$ & $\mathrm{kg} / \mathrm{s}$ & $\mathrm{kg} / \mathrm{s}$ & $\mathrm{N} / \mathrm{m}$ & $\mathrm{N} / \mathrm{m}$ \\
\hline Value & 128 & 3.738 & 7.476 & 2885.48 & 206.87 & $40 \times 10^{6}$ & $1.9 \times 10^{6}$ \\
\hline Parameter & $c$ & $m_{\mathrm{d}}$ & $r_{\mathrm{s}}$ & $l_{\mathrm{r}}$ & $e$ & $l_{\mathrm{s}}$ & $\gamma$ \\
\hline Dimension & $\mathrm{N} / \mathrm{m}$ & $\mathrm{kg}$ & $\mathrm{m}$ & $\mathrm{m}$ & $\mathrm{m}$ & $\mathrm{m}$ & $\mathrm{rad}$ \\
\hline Value & $1.8 \times 10^{6}$ & 0.01 & 0.270 & 0.6 & $5 \times 10^{-6}$ & 0.340 & 1.6 \\
\hline Parameter & $l$ & \multicolumn{2}{|c|}{$r_{\mathrm{r}}$} & $a_{2}$ & \multicolumn{2}{|c|}{$a_{3}$} & $\delta$ \\
\hline Dimension & $\mathrm{m}$ & \multicolumn{2}{|c|}{$\mathrm{m}$} & $\mathrm{N}$ & \multicolumn{2}{|c|}{$\mathrm{Nm}$} & $\mathrm{rad}$ \\
\hline Value & 0.790 & \multicolumn{2}{|c|}{0.0225} & $12.515 \times 10^{6}$ & \multicolumn{2}{|c|}{$41.257 \times 10^{5}$} & 0.01 \\
\hline Parameter & $\overline{\overline{l_{1}}}$ & \multicolumn{2}{|c|}{$\bar{a} a_{1}$} & $\bar{a}$ & \multicolumn{2}{|l|}{$b$} & $\overline{\Delta^{2}}$ \\
\hline Dimension & $\mathrm{m}$ & \multicolumn{2}{|c|}{$\mathrm{N} / \mathrm{m}$} & $\mathrm{m}^{2} \mathrm{~kg}^{2} / \mathrm{N}^{2}$ & \multicolumn{2}{|l|}{$\mathrm{mkg} / \mathrm{N}$} & \\
\hline Value & 0.37 & \multicolumn{2}{|c|}{$42.663 \times 10^{6}$} & $1.5 \times 10^{-11}$ & \multicolumn{2}{|l|}{$2.2 \times 10^{-5}$} & $5.098 \times 10^{-14}$ \\
\hline
\end{tabular}
the bearing. Simulation results are presented in Fig. 5.
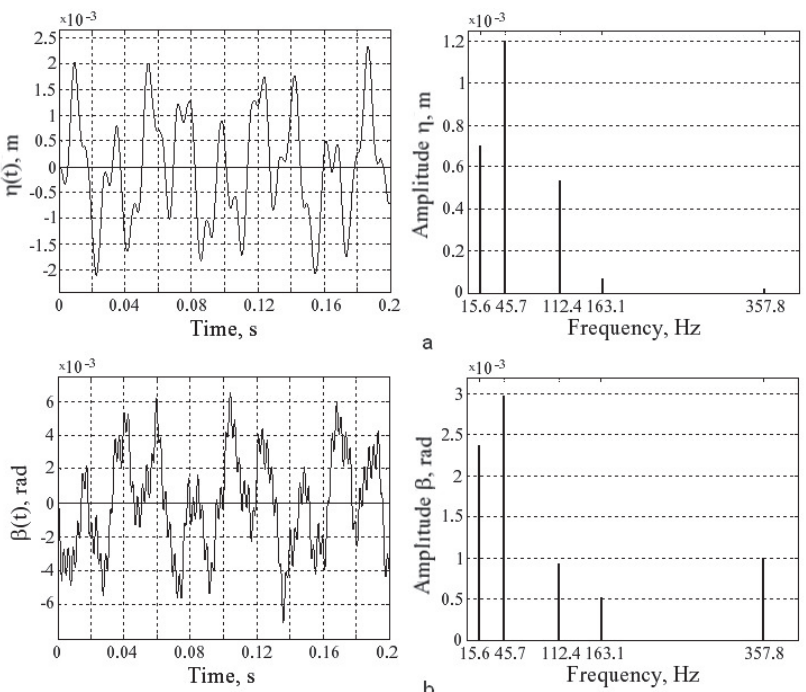

Figure 5 Vibration amplitude-time response and amplitude-frequency spectrum of the rotor: (a) - along $\eta$ coordinate axis, (b) - for the angle $\beta$

Table 1 Simulation data 

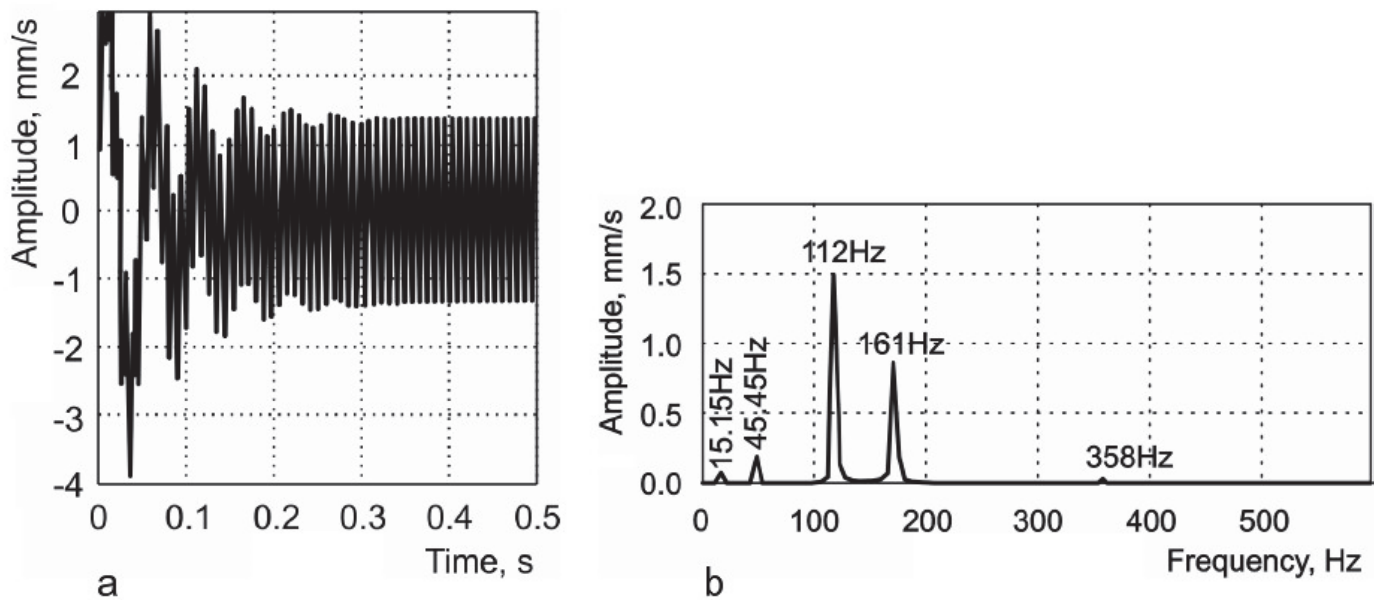

Figure 6 (a) vibration velocity amplitude versus time plot and (b) amplitude-frequency spectrum obtained for the outer ring of rotor bearing
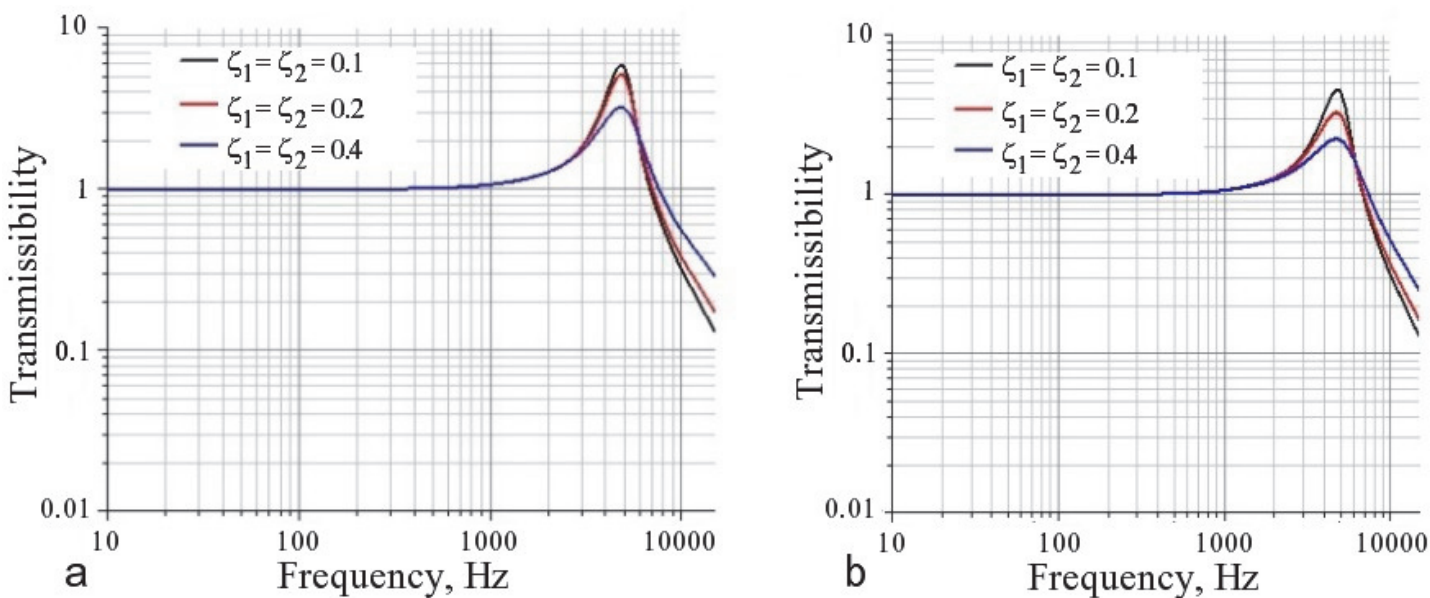

Figure 7 Vibration transmissibility curves obtained for different values of dimensionless damping coefficient $\xi$ : (a) - viscoelastic damping, (b) - viscoelastic and hysteretic damping

Amplitude-frequency responses (Fig. 5) show that rotor rotates with non-resonant frequency. Low frequency vibrations are caused by the electromotor, step-up gear and bearing system.

Vibration velocity amplitude versus time plot and amplitude-frequency spectrum of outer ring of the bearing were obtained using data presented in Tab. 1. Simulation results are presented in Fig. 6. It can be seen from Fig. 6a that the mechanism is excited after the electromotor starts and the amplitude is increased during $0.2 \mathrm{~s}$ period of time. Then velocity amplitude is stabilized at some level. The spectral density represents the characteristic frequencies of the machine, i.e. of electromotor, rotor, bearing cage and other components. From Fig. 6b, it can be seen that if excitation frequency is $112 \mathrm{~Hz}$, the system vibrates at four frequencies: $15.15 \mathrm{~Hz}, 45.45 \mathrm{~Hz}, 161 \mathrm{~Hz}$ and $358 \mathrm{~Hz}$.

Vibration transmissibility [27] calculation results are presented in Fig. 7. The obtained results show that in case of viscoelastic damping resonance starts at $796.2 \mathrm{~Hz}$ frequency. In case of hysteretic damping resonance appears at $716.6 \mathrm{~Hz}$ frequency. The effective damping is achieved in the first case at $1273.9 \mathrm{~Hz}$ frequency, in the second case - at $1194.3 \mathrm{~Hz}$, i.e. at the high frequencies. This requires experimental verification, because these frequencies are the doubled frequencies excited by bearing balls rolling between an outer and an inner ring. The low frequency vibrations are insufficiently suppressed (Fig. 7).

\subsection{Experimental Results}

After a comparison of theoretical [28, 29] and experimental (obtained from measurements (Fig. 8)) amplitude-frequency spectrum, the most damaged bearing elements can be identified. According to the vibration velocity it is possible to assess the level of damage. It can be seen that theoretical frequencies are not sufficiently distinguished in the experimentally obtained spectrum (Fig. 8), because theoretical calculations were performed for a single-row bearing. Investigated bearing is a doublerow bearing (Fig. 1), therefore frequencies shifted to the higher frequency region or other frequency combinations were generated. In addition, other components of separator, such as electromotor and step-up gear affect the results of measurements.

It can be stated that the rolling elements and the outer ring are the most damaged parts of the bearing. However, during normal operation of the centrifugal milk separator, direct measurements of vibrations of the bearing are not possible. Therefore, vibrations of the housing which is connected to the vibration damper have to be measured. Results are presented in Fig. 9.

After analysis of amplitude-frequency spectra presented in Figs. 8 and 9, it can be concluded that bearing vibration level and frequency distribution are affected by vibration damping system. Therefore, by measuring vibrations transmitted through the vibration damping 
system, it is not possible to diagnose properly the state of the bearing-rotor system. It is therefore necessary to establish the influence of the vibration damping system on the measurement results and create a new rotor bearing fault diagnosis technique.

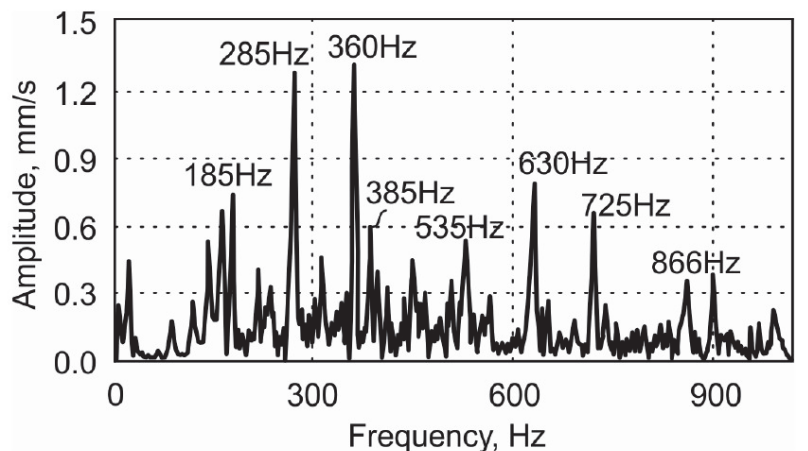

Figure 8 Experimental vibration velocity amplitude-frequency spectrum (bearing vibration measurement results)

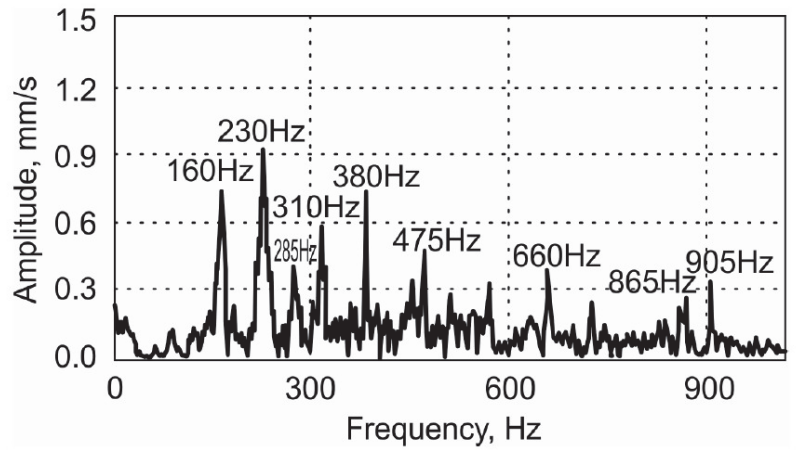

Figure 9 Experimental vibration velocity amplitude-frequency spectrum (housing vibration measurement results)

\section{CONCLUSIONS}

Obtained results demonstrated the problems that occur when results of indirect vibration measurements are used to detect faults in damped bearing-rotor systems.

Simulation of the milk flow inside the centrifugal separator has shown that rotational milk motion causes the centrifugal force, which in conjunction with rotor unbalance force is the main source of vibrations acting on the bearings and vibration dampers. However, calculated vibration transmissibility of vibration dampers shows that the resonant frequency is higher than the rotational frequency of the rotor and is close to the doubled frequencies excited by the balls rolling between an inner and an outer ring of the bearing.

It is established that critical speed of the rotor is close to its double rotational speed, but it does not exceed the resonant frequency of the system. Simulation has shown the significant influence of stiffness of components of vibration damping system on the bearing vibration amplitude. Vibration amplitude increases four times while the stiffness of components of vibration damping system decreases twice.

Experimental studies have shown that vibration measurements of the housing of centrifugal milk separator are little informative at low and medium frequencies. Therefore, new fault diagnosis techniques are needed for machines of this type.

\section{REFERENCES}

[1] Vekteris, V., Trumpa, A., Turla, V., Šešok, N., Iljin, I., Mokšin, V., Kilikevičius, A., Jakštas, A., \& Kleiza, J. (2017). An investigation into fault diagnosis in a rotor-bearing system with dampers used in centrifugal milk separators. Transactions of FAMENA, 41(2), 77-86. https://doi.org/10.21278/TOF.41207

[2] Trumpa, A., Vekteris, V., Mokšin, V., \& Kilikevičius, A. (2018). Fault diagnostic system for centrifugal milk separator's rotor bearings with vibration isolators. Tehnicki Vjesnik - Technical Gazette, 25(4), 986-990. https://doi.org/10.17559/TV-20160501130126

[3] Xu, M. \& Marangoni, R. D. (1994). Vibration analysis of a motor-flexible coupling-rotor system subject to misalignment and unbalance. Part I: Theoretical model and analysis. Journal of Sound and Vibration, 176(5), 663-679. https://doi.org/10.1006/jsvi.1994.1405

[4] Chow, E. Y.\& Willsky, A. S. (1984). Analytical redundancy and the design of robust detection systems. IEEE Transactions on Automatic Control, 29(7), 603-614. https://doi.org/10.1109/TAC.1984.1103593

[5] Gertler, J. J. (1993). Residual generation in model based fault diagnosis. Control Theory and Advanced Technology, 9(1), 259-285.

[6] Isermann, R. (1995). Model based fault detection and diagnosis methods. Proceedings of 1995 American Control Conference, Seattle, USA, 1995, 1605-1609.

[7] Jalan, A. Kr. \& Mohanty, A. R. (2009). Model based fault diagnosis of a rotor-bearing system for misalignment and unbalance under steady-state condition. Journal of Sound and Vibration, 327(3-5), 604-622. https://doi.org/10.1016/j.jsv.2009.07.014

[8] Harsha, S. P. (2005). Nonlinear dynamic analysis of an unbalanced rotor supported by roller bearing. Chaos, Solitons \& Fractals, 26(1), 47-66. https://doi.org/10.1016/j.chaos.2004.12.014

[9] Tiwari, M., Gupta, K., \& Prakash, O. (2000). Dynamic response of an unbalanced rotor supported on ball bearings. Journal of Sound and Vibration, 238(5), 757-779. https://doi.org/10.1006/jsvi.1999.3108

[10] Upadhyay, S. H., Harsha, S. P., \& Jain, S. C. (2010). Analysis of nonlinear phenomena in high speed ball bearings due to radial clearance and unbalanced rotor effects. Journal of Vibration and Control, 16(1), 65-88. https://doi.org/10.1177/1077546309104188

[11] Sunnersjo, C. S. (1978). Varying compliance vibrations of rolling bearings. Journal of Sound and Vibration, 58(3), 363373. https://doi.org/10.1016/S0022-460X(78)80044-3

[12] Fukata, S., Gad, E. H., Kondou, T. A., Ayabe, T., \& Tamura, H. (1985). On the radial vibrations of ball bearings: Computer simulation. Bulletin of the JSME, 28(239), 899904. https://doi.org/10.1299/jsme1958.28.899

[13] Perret, H. (1950). Elastiches piel schwingung en constant belaster walzlger. Werkstatt und Betrieb, 3, 354-358.

[14] Meldau, E. (1951). Die bewegung der achse von walzlagern bei geringen drehzahlen. Werkstatt und Betrieb, 7, 308-313.

[15] Mevel, B. \& Guyader, J. L. (2008). Experiments on routes to chaos in ball bearings. Journal of Sound and Vibration, 318(3), 549-564. https://doi.org/10.1016/j.jsv.2008.04.024

[16] Datta, J. \& Farhang, K. (1997). A nonlinear model for structural vibrations in rolling element bearings. Part I: Derivation of governing equations. Journal of Tribology, 119(1), 126-131. https://doi.org/10.1115/1.2832445

[17] Tiwari, M., Gupta, K., \& Prakash, O. (2000). Effect of radial internal clearance of a ball bearing on the dynamics of a balanced, horizontal rotor. Journal of Sound and Vibration, 238(5), 723-756. https://doi.org/10.1006/jsvi.1999.3109

[18] Harsha, S. P., Sandeep, K., \& Prakash, R. (2003). The effect of speed of balanced rotor on nonlinear vibrations associated 
with ball bearings. International Journal of Mechanical Sciences, 45(4), 725-740. https://doi.org/10.1016/S0020-7403(03)00064-X

[19] Harsha, S. P. (2005). Non-linear dynamic response of a balanced rotor supported on rolling element bearings. Mechanical Systems and Signal Processing, 19(3), 551-578. https://doi.org/10.1016/j.ymssp.2004.04.002

[20] Harsha, S. P. (2006). Nonlinear dynamic analysis of a highspeed rotor supported by rolling element bearings. Journal of Sound and Vibration, 290(1-2), 65-100. https://doi.org/10.1016/j.jsv.2005.03.008

[21] Yadav, H. K., Upadhyay, S. H., \& Harsha, S. P. (2013). Study of effect of unbalanced forces for high speed rotor. Procedia Engineering, 64, 593-602. https://doi.org/10.1016/.proeng.2013.09.134

[22] Bai, C., Zhang, H., \& Xu, Q. (2013). Subharmonic resonance of a symmetric ball bearing-rotor system. International Journal of Non-Linear Mechanics, 50, 1-10. https://doi.org/10.1016/j.jinonlinmec.2012.11.002

[23] Kumar, H., Sugumaran, V., \& Amarnath, M. (2016). Fault diagnosis of bearings through sound signal using statistical features and Bayes classifier. Journal of Vibration Engineering and Technologies, 4(2), 87-96.

[24] SolidWorks Flow Simulation Technical Reference. Dassault Systemes SolidWorks Corp., 2009.

[25] Vekteris, V., Styra, A., Mokšin, V., Grinbergienè, I., Jurevičius, M., Turla, V., \& Mester, G. (2018). Numerical simulation of a pulsating flow generated in an ejector. Transactions of FAMENA, 42(2), 97-107. https://doi.org/10.21278/TOF.42208

[26] Koritysskiy, Y. A. (1973).Vibrations in Textile Machines. Moscow: Mashinostroyenie (In Russian).

[27] Trumpa, A. \& Vekteris, V. (2006). Diagnostics of the mechatronical systems with vibration insulation. Solid State Phenomena, 113, 371-374. https://doi.org/10.4028/www.scientific.net/SSP.113.371

[28] Tandon, N. \& Choudhury, A. (1999). A review of vibration and acoustic measurement methods for the detection of defects in rolling element bearings. Tribology International, 32(8), 469-480. https://doi.org/10.1016/S0301-679X(99)00077-8

[29] Saidi, L., Ben Ali, J., \& Fnaiech, F. (2015). Application of higher order spectral features and support vector machines for bearing faults classification. ISA Transactions, 54, 193206. https://doi.org/10.1016/j.isatra.2014.08.007
Igor ILJIN, Associate Professor, PhD

Vilnius Gediminas Technical University,

Department of Mechatronics, Robotics and Digital Manufacturing,

J. Basanavičiaus 28, 03224 Vilnius, Lithuania

E-mail: igor.iljin@vilniustech.It

\section{Contact information}

Andrius TRUMPA, Lecturer, PhD

Vilnius Gediminas Technical University,

Department of Mechanical and Material Engineering,

J. Basanavičiaus 28, 03224 Vilnius, Lithuania

E-mail: andrius.trumpa@vilniustech.lt

Vadim MOKŠIN, Full Professor, PhD

(Corresponding author)

Vilnius Gediminas Technical University,

Department of Mechanical and Material Engineering,

J. Basanavičiaus 28, 03224 Vilnius, Lithuania

E-mail: vadim.moksin@vilniustech.It

Vytautas TURLA, Full Professor, PhD

Vilnius Gediminas Technical University,

Department of Mechatronics, Robotics and Digital Manufacturing,

J. Basanavičiaus 28, 03224 Vilnius, Lithuania

E-mail: vytautas.turla@vilniustech.It

Nikolaj ŠEŠOK, Associate Professor, PhD

Vilnius Gediminas Technical University,

Department of Mechatronics, Robotics and Digital Manufacturing,

J. Basanavičiaus 28, 03224 Vilnius, Lithuania

E-mail: nikolaj.sesok@vilniustech.It 